\title{
Possibilistic uncertainty analysis of a conceptual model of snowmelt runoff
}

\section{A. P. Jacquin}

Facultad de Ingeniería, Pontificia Universidad Católica de Valparaíso, Av. Brasil 2147, Valparaíso, Chile

Received: 11 March 2010 - Published in Hydrol. Earth Syst. Sci. Discuss.: 24 March 2010

Revised: 29 July 2010 - Accepted: 20 August 2010 - Published: 30 August 2010

\begin{abstract}
This study presents the analysis of predictive uncertainty of a conceptual type snowmelt runoff model. The method applied uses possibilistic rather than probabilistic calculus for the evaluation of predictive uncertainty. Possibility theory is an information theory meant to model uncertainties caused by imprecise or incomplete knowledge about a real system rather than by randomness. A snow dominated catchment in the Chilean Andes is used as case study. Predictive uncertainty arising from parameter uncertainties of the watershed model is assessed. Model performance is evaluated according to several criteria, in order to define the possibility distribution of the parameter vector. The plausibility of the simulated glacier mass balance and snow cover are used for further constraining the model representations. Possibility distributions of the discharge estimates and prediction uncertainty bounds are subsequently derived. The results of the study indicate that the use of additional information allows a reduction of predictive uncertainty. In particular, the assessment of the simulated glacier mass balance and snow cover helps to reduce the width of the uncertainty bounds without a significant increment in the number of unbounded observations.
\end{abstract}

\section{Introduction}

Uncertainty can be defined as the lack of necessary information to "quantitatively and qualitatively ... describe, prescribe, or predict deterministically and numerically a system, its behaviour or other characteristica" (Zimmermann, 2001). Even the most complex model of a real system necessarily involves a series of assumptions and approximations, which

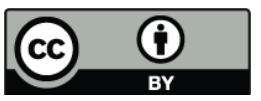

Correspondence to: A. P. Jacquin (alexandra.jacquin@ucv.cl) are necessary to compensate for our incomplete understanding of the real world. Unfortunately, the question of whether or not these assumptions and approximations are justifiable can hardly be answered with absolute certitude, which implies that the reliability of the model is ultimately uncertain. Uncertainties arising in engineering modelling can be classified into two groups (Ferson and Ginzburg, 1996; Ayyub and Klir, 2006): aleatory and epistemic uncertainties. Aleatory uncertainties originate from the inherent variability of some phenomena that are perceived as being governed by underlying stochastic processes. The fact that this variability is treated as an inherent property of the real system implies that aleatory uncertainties cannot be reduced by improving the knowledge available to the modeller. Aleatory uncertainties can be dealt with using an objective (frequentist) probabilistic approach. On the other hand, epistemic uncertainties are those arising from the incompleteness of the information about the real system that is available to the modeller. Given that these uncertainties are related to the state of knowledge, they can sometimes be reduced by improving it. Subjective (Bayesian) probability approaches and non-probabilistic frameworks are suitable for analysing uncertainties that do not have a stochastic nature.

Watershed models attempt to simulate the complex hydrological processes that lead to the transformation of precipitation into runoff. The sources contributing to the overall uncertainty in the discharge estimates provided by these models can be grouped in three categories, namely, data uncertainty, model structure uncertainty and parameter uncertainty (Bates and Townley, 1988; Lei and Shilling, 1996). Variations in natural phenomena (e.g. spatial and temporal variation of rainfall), are usually treated as having an aleatory nature. By contrast, other uncertainties affecting hydrological data (e.g. adequacy of the rainfall network distribution, validity of the flow rating curves, etc.), model structure uncertainty and parameter uncertainty have an epistemic rather

Published by Copernicus Publications on behalf of the European Geosciences Union. 
than a stochastic nature. It is widely recognized that, because of these uncertainties and their implications, choosing a single model representation (i.e. a combination of a model structure and a parameter set) for simulating runoff generation in a particular catchment is a common practice that is not necessarily supported by evidence (see e.g. Beven, 2006; Wagener et al., 2003). Most likely, there may be many acceptable model representations whose rejection cannot be justified, considering the always limited information available to the modeller. This non-uniqueness of the model representations, sometimes called equifinality in the hydrological literature (Beven and Freer, 2001; Beven, 2006), is a problem that has long been recognized in the context of linear systems theory (see e.g. Cheng, 1959; Zadeh and Desoer, 1963). Nevertheless, reality outside the scientific context is that field practitioners rarely include an analysis of predictive uncertainty when applying watershed models in water resources studies. Moreover, there is an important number of water researchers still unconvinced that uncertainty analysis should necessarily be part of the modelling process (Pappenberger and Beven, 2006).

In spite of the reluctance of some scientists, hydrologists have been very active in developing methods for uncertainty analysis. Extensive reviews on this topic have been presented in the literature (e.g. Matott et al., 2009; Montanari et al., 2009). Probabilistic approaches used in hydrology include variance propagation (e.g. Kuczera, 1988; Bates and Townley, 1988), Monte Carlo sampling coupled with frequency analysis (e.g. Bates and Townley, 1988; Yu et al., 2001; Thorsen et al., 2001; Zehe and Blöschl, 2004; Arnold et al., 2009), Bayesian analysis (e.g. Beven and Binley, 1992; Romanowicz et al., 1994; Thiemann et al., 2001; Misirli et al., 2003; Engeland et al., 2005; Rojas et al., 2010; Renard et al., 2010) and imprecise probabilities (Hall, 2006; Ghosh and Mujumdar, 2009; Nijssen et al., 2009). Non-probabilistic methods found in the hydrological literature include those based on fuzzy sets theory (e.g. Dou et al., 1997; Seibert, 1997; Freissinet et al., 1999; Özelkan and Duckstein, 2001; Bárdossy et al., 2006; Zhang et al., 2009) and possibility theory (Martin-Clouaire et al., 2000; Verhoest et al., 2007; Mujumdar and Ghosh, 2008).

In this study, predictive uncertainty of a conceptual type snowmelt runoff model is analysed using a methodology recently proposed in the context of watershed modelling (Jacquin and Shamdeldin, 2007). This method, based on possibility theory (Zadeh, 1978, 1981), explicitly accounts for the problem of the non-uniqueness of model representations. So far, this possibilistic method has been tested in very few cases, all of which correspond to watershed models without a snowmelt runoff component (Jacquin and Shamdeldin, 2007, 2009). The applicability of the method to other model structures has not been explored. In particular, the present study is the first attempt to investigate the applicability of the method in snowmelt runoff modelling. Furthermore, the effect of several subjective choices made during the inference process, such as the possibility level at which uncertainty bounds are derived and the criteria used for the evaluation of model plausibility, have not been analysed.

\section{Possibility theory}

\subsection{Introductory remarks}

Possibility theory is an information theory that was first proposed as an extension of fuzzy sets theory for representing vague linguistic information (Zadeh, 1978, 1981). Since then, possibility theory has been further developed into an independent information theory. Possibility theory provides an appropriate framework for the analysis of uncertainties with an epistemic nature, such as those arising from model uncertainty and parameter uncertainty in watershed modelling. Even though possibility theory has been available for decades, the application of possibilistic calculus in uncertainty analysis is still very rare in hydrology. Examples of this line of work include mappings of soil hydrological properties (Martin-Clouaire et al., 2000), soil moisture retrieval from radar remote sensing data (Verhoest et al., 2007), and modelling uncertainties affecting General Circulation Models and future scenarios in climate change impact evaluation (Mujumdar and Ghosh, 2008).

The word possibility can be interpreted as either feasibility (i.e. degree of ease in a physical sense) or plausibility (Dubois and Prade, 1998). In this work, the word possibility is interpreted in the epistemic sense of plausibility, understood as the degree to which the occurrence of an event would not be surprising. The potential surprise of an event reflects the extent to which the evidence available is in contradiction with its occurrence (Dubois and Prade, 1998). The remainder of this section provides a brief introduction to possibility theory, but more complete discussions on this subject can be found in the literature (e.g. Klir and Folger, 1992; Dubois and Prade, 1998; Wolkenhauer, 1998).

\subsection{Possibility and necessity measures}

Possibility theory describes partial belief in terms of possibility and necessity measures, which are dual set functions defined on the power set (i.e. the collection of all possible subsets) of the universe of discourse $U$. Possibility and necessity measures are special cases of plausibility and belief measures, respectively, as defined by the theory of evidence of Dempster-Shafer (Shafer, 1976; Dempster, 1967).

A normal possibility measure $\Pi$ is a mapping from the power set $2^{U}$ to the interval [0,1], such that

$$
\begin{aligned}
& \Pi(\Phi)=0, \\
& \Pi(U)=1, \text { and } \\
& \Pi(S \cup R)=\max \{\Pi(S), \Pi(R)\},
\end{aligned}
$$


where $S$ and $R$ are subsets of $U$. If the universe of discourse $U$ is infinite (e.g. an interval of the real line), the relationship expressed in Eq. (3) must be extended to any family of subsets $S_{i}$, as expressed by

$$
\Pi\left(\cup_{i} S_{i}\right)=\sup _{i} \Pi\left(S_{i}\right) .
$$

The possibility degree $\Pi(S)$ estimates the lack of contradiction between the evidence and the statement " $\boldsymbol{X} \in S$ " (i.e. " $\boldsymbol{X}$ belongs to $S$ "), where $\boldsymbol{X}$ is a variable or vector of variables from the universe of discourse $U$, and $S$ is a subset of $U$. Hence, the possibility degree $\Pi(S)$ indicates the plausibility of the statement " $\boldsymbol{X} \in S$ ", given the knowledge available about $\boldsymbol{X}$ (Dubois and Prade, 1998). A possibility degree $\Pi(S)=1$ indicates that " $\boldsymbol{X} \in S$ " is totally compatible with the evidence (thus, fully possible), while a possibility degree $\Pi(S)=0$ indicates that " $\boldsymbol{X} \in S$ " is in total contradiction with the knowledge available about $\boldsymbol{X}$ (thus, impossible). By contrast, the necessity degree of a subset $S$ (denoted by $N(S)$ ) estimates the degree of certainty in that " $\boldsymbol{X} \in S$ ", as supported by the evidence. Representing complementary aspects of partial belief, dual possibility and necessity measures are related according to

$N(S)=1-\Pi\left(S^{\mathfrak{c}}\right)$,

where $S^{\mathrm{c}}$ represents the complement of the subset $S$. Thus, the necessity degree $N(S)$ represents the lack of plausibility of $S^{\mathrm{c}}$. A necessity degree $N(S)=1$ indicates that there is total certainty in that " $\boldsymbol{X} \in S$ ", while a necessity degree $N(S)=0$ indicates there is no certainty in that " $\boldsymbol{X} \in S$ ", although this could still be possible with $\Pi(S)>0$.

\subsection{Possibility distributions}

A possibility distribution $\pi$ is a mapping from the universe of discourse $U$ to the interval $[0,1]$. The possibility value $\pi(\boldsymbol{x})$ represents the plausibility that $\boldsymbol{X}$ takes the value $\boldsymbol{X}$ (i.e. $\boldsymbol{X}=\boldsymbol{x}$ ), given the knowledge available about $X$. For example, Fig. 1 shows a possibility distribution of the variable $X$, in which the possibility value of $u_{1}$ is $\pi\left(u_{1}\right)=\alpha_{1}$, the possibility value of $u_{2}$ is $\pi\left(u_{2}\right)=\alpha_{2}$, etc. A possibility distribution $\pi$ is said to be normal if

$$
\sup _{x \in U} \pi(x)=1
$$

as in the case of the possibility distribution depicted in Fig. 1 . In this situation, a possibility value $\pi(x)=1$ indicates that $\boldsymbol{X}=\boldsymbol{x}$ is totally compatible with the knowledge available about $\boldsymbol{X}$; a possibility value $\pi(\boldsymbol{x})=0$ arises if $\boldsymbol{X}=\boldsymbol{x}$ is in total contradiction with the evidence. Every possibility possibility distribution $\pi$ induces a possibility measure $\Pi$, given by

$$
\Pi(S)=\sup _{x \in S} \pi(\boldsymbol{x})
$$

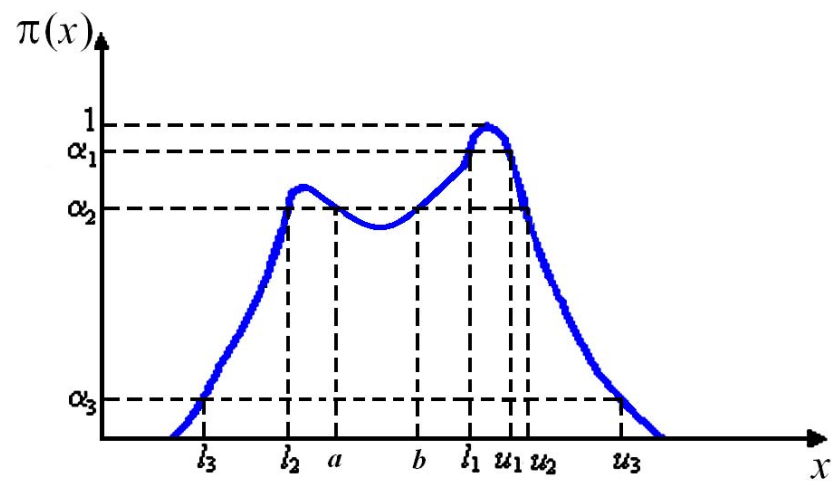

Fig. 1. Example of a normal possibility distribution with two local maxima.

for every subset $S \subseteq U$. In the example shown in Fig. 1, the possibility degree of the subset $R=\left[l_{1}, u_{1}\right]$ is $\Pi(R)=1$, because a value $x_{0} \in\left[l_{1}, u_{1}\right]$ exists such that $\pi\left(x_{0}\right)=1$; similarly, application of Eq. (5) yields the conclusion that the necessity degree of $R=\left[l_{1}, u_{1}\right]$ is $N(R)=1-\alpha_{1}$.

In their classical interpretation, possibility distributions are seen as being induced by fuzzy sets describing vaguely defined concepts (Zadeh, 1978, 1981). More concretely, every preposition of the form " $\boldsymbol{X}$ is $A$ ", where $A$ is a fuzzy set in $U$, induces a possibility distribution $\pi$ whose possibility values are given by

$\pi(\boldsymbol{x})=\mu_{A}(\boldsymbol{x})$,

where $\mu_{A}$ is the membership function of the fuzzy set $A$. Another interpretation of possibility distributions relates them with likelihood functions of the type used in statistical inference. In particular. it has been suggested that maximum likelihood methods for hypothesis testing treat likelihood functions as possibility distributions of the uncertain variables (Dubois and Prade, 1993; Dubois et al., 1997).

\section{$2.4 \alpha$-cuts and strong $\alpha$-cuts}

The $\alpha$-cut of a possibility distribution $\pi$ is the set of all $\boldsymbol{x} \in U$ with possibility values $\pi(\boldsymbol{x})$ greater than or equal to $\alpha$, that is

$C^{\alpha}=\{\boldsymbol{x} / \pi(\boldsymbol{x}) \geq \alpha\}$,

for every $\alpha \in[0,1]$. For example, the $\alpha_{1}$-cut of the possibility distribution shown in Fig. 1 is given by the interval $\left[l_{1}, u_{1}\right]$ and the $\alpha_{3}$-cut is given by the interval $\left[l_{3}, u_{3}\right]$, while the $\alpha_{2}$-cut corresponds to the union of intervals $\left[l_{2}, a\right]$ and $\left[b, u_{2}\right]$. Similarly, the strong $\alpha$-cut of a possibility distribution $\pi$ corresponds to the set of all $x \in U$ with possibility values $\pi(\boldsymbol{x})$ strictly greater than $\alpha$, that is

$C^{\prime \alpha}=\{\boldsymbol{x} / \pi(\boldsymbol{x})>\alpha\}$,

for every $\alpha \in[0,1]$. In the example shown in Fig. 1, the strong $\alpha_{1}$-cut is is given by the interval $] l_{1}, u_{1}[$ and the strong 
$\alpha_{3}$-cut is is given by the interval $] l_{3}, u_{3}\left[\right.$, while the strong $\alpha_{2}$ cut corresponds to the union of intervals $] l_{2}, a[$ and $] b, u_{2}[$. Equations (9) and (10) imply that the possibility degree of any $\alpha$-cut or strong $\alpha$-cut with $\alpha \in[0,1$ [ is always equal to unity, because the value $\boldsymbol{x}_{0}$ such that $\pi\left(\boldsymbol{x}_{0}\right)=1$ is necessarily inside the $\alpha$-cut or strong $\alpha$-cut (see e.g. Fig. 1). According to Eq. (5), the necessity degree of an $\alpha$-cut or strong $\alpha$-cut is $1-\alpha$ for any $\alpha \in[0,1[$.

If a continuous possibility distribution $\pi$ defined on a universe of discourse that is an interval of the real line is unimodal, then its $\alpha$-cuts and strong $\alpha$-cuts are intervals. If the possibility distribution $\pi$ has several local maxima, as in the example shown in Fig. 1, this property does not hold. In this situation, only possibility levels that are higher than all local maxima different from the global maximum (e.g. the possibility level $\alpha_{1}$ in Fig. 1), and possibility levels that are lower than all local minima (e.g. the possibility level $\alpha_{3}$ in Fig. 1), define $\alpha$-cuts and strong $\alpha$-cuts that are intervals. In a possibility distribution with several local maxima, a possibility level $\alpha$ that does not fulfil these requirements defines an $\alpha$ cut and a strong $\alpha$-cut that are given by the union of two or more intervals (e.g. the possibility level $\alpha_{2}$ in Fig. 1).

\subsection{The Extension Principle}

The Extension Principle, first proposed in the context of fuzzy sets theory (Zadeh, 1965), allows to determine the possibility distribution of a variable $Y$ having a functional relation with a variable or vector $\boldsymbol{X}$ (i.e. $Y=f(\boldsymbol{X})$ ) whose possibility distribution $\pi$ is known. By virtue of the Extension Principle, the possibility distribution of $Y$ is given by (Zadeh, 1981)

$\pi_{Y}(y)=\left\{\begin{array}{l}\max _{\boldsymbol{x}, f(\boldsymbol{x})=y} \pi(\boldsymbol{x}) \\ 0, \text { if } f(\boldsymbol{x}) \neq y \text { for all } \boldsymbol{x}\end{array}\right.$,

where $y$ is a possible value of the variable $Y$. Equation (11) shows that the possibility value assigned to $y$ is the maximum possibility value encountered among all the values $x$ such that $y=f(\boldsymbol{x})$. If no value $\boldsymbol{x}$ exists such that $y=f(\boldsymbol{x})$, then the possibility value $\pi_{Y}(y)$ is equal to zero.

\subsection{Relationship between possibility theory and probability theory}

Quantitative possibility theory provides a framework for representing degrees of belief that is alternative to the subjective view of probability theory (Dubois et al., 2008). In this context, possibility theory and probability theory should be seen as complementary rather than competitive, as they are intended to represent different levels of information (Klir and Folger, 1992; Dubois and Prade, 1993; Ross et al., 2002). There are several manners to interpret the relationship between possibilities and probabilities (see e.g. Zadeh, 1978; Klir and Folger, 1992; Dubois and Prade, 1993, 1998).
Perhaps the most intuitive notion is that possibility degrees provide an upper bound for probability degrees, in the sense that something must be possible before being probable (Zadeh, 1978, 1981). Formally, this interpretation is justified by the fact that a possibility measure can be seen as the upper envelope of a family of probability measures (see e.g. Dubois and Prade, 1992, 1993; Baudrit and Dubois, 2005). Hence, a possibility measure provides a less specific representation of uncertainty than that encoded by a single probability measure (Dubois and Prade, 1993). In addition to this, numerous studies have proposed methods for probability-possibility transformations (Civanlar and Trussel, 1986; Dubois and Prade, 1986, 1993, 1998; Dubois et al., 2004; Klir and Geer, 1993). Finally, other studies have investigated the relationship between possibility distributions and confidence intervals (Dubois et al., 2004; Baudrit and Dubois, 2006).

\section{Possibilistic method for uncertainty analysis in watershed modelling}

\subsection{Context}

In recent years, a possibilistic uncertainty analysis method originally inspired by the widely known Generalized Likelihood Uncertainty Estimation (GLUE) methodology (Beven and Binley, 1992) was proposed (Jacquin and Shamseldin, 2007). The difference between both methods is that GLUE follows a subjective probabilistic scheme, but the method applied herein uses possibilistic calculus in order to assess predictive uncertainty. More concretely, the derivation of uncertainty bounds within the GLUE methodology relies on the calculation of prediction quantiles from the likelihood weights of the model predictions at each time step, while the possibilistic method applies the Extension Principle (Zadeh, 1981) in order to estimate the possibility distribution of the discharge predictions from the information on the possibility values of the model realizations in a sample. A discussion on the advantages of the possibilistic method with respect to the probabilistic approach of GLUE can be found in the paper where the possibilistic method was first presented (Jacquin and Shamseldin, 2007). A brief description of the possibilistic method is given in what follows.

\subsection{Generation of a sample of model realizations}

Similar to the GLUE methodology, the possibilistic method also allows the estimation of predictive uncertainty arising from model structure and parameter uncertainties using a sample of model realizations. However, the following discussion assumes that a single model structure is being considered and that only parameter uncertainty is being analysed. In this case, the sample of model realizations is obtained by generating a large sample of the parameter vector $\boldsymbol{\theta}=\left\{\theta_{1}, \theta_{2}, \ldots \theta_{n}\right\}$, where each component of this vector represents one of the model parameters and $n$ is the total number 
of parameters involved in the model structure. The initial possibility distribution of the parameter vector, $\pi_{\text {initial }}$, is defined from the prior knowledge available about the regions of the parameter space that are associated with good model realizations. The initial possibility values of the parameter vectors in the sample, $\pi_{\text {initial }}(\boldsymbol{\theta})$, are subsequently calculated.

When the possibilistic method applied herein was first presented, the use of a random sampling strategy with uniform probability distributions was proposed in order to simplify the calculations (Jacquin and Shamseldin, 2007). Acknowledging the fact that other sampling strategies were also possible, the former authors also pointed out that the results of the method do not directly depend on the probability distribution that is used for generating the sample. Other sampling strategies, such as a systematic sampling scheme, can also be used. All that is required is a sample of the parameter vector that provides a sufficient exploration of the parameter space, in order to empirically derive the possibility distribution of the parameter vector by evaluation of the goodness of fit of the model realizations.

\subsection{Possibility distribution of the parameter vector}

The goodness of fit of the model realizations is further evaluated using a chosen measure of model performance together with a model rejection criterion. The values of this measure of model performance are used for deriving the possibility distribution of the parameter vector, $\pi$. Parameter vectors achieving possibility values $\pi(\boldsymbol{\theta})=1$ are those associated with the model realizations providing the best fit to the observations, according to the chosen measure of model performance. Parameter vectors associated with model realizations deemed inacceptable by the model rejection criterion are assigned possibility values $\pi(\boldsymbol{\theta})=0$. The possibility distribution $\pi$ is combined with the initial possibility distribution $\pi_{\text {initial }}$ using the normalized product conjunction rule. The aggregate possibility distribution of the parameter vector is thus given by

$\pi_{\text {agg }}(\boldsymbol{\theta})=\frac{\pi_{\text {initial }}(\boldsymbol{\theta}) \cdot \pi(\boldsymbol{\theta})}{\max _{\boldsymbol{\theta}}\left\{\pi_{\text {initial }}(\boldsymbol{\theta}) \cdot \pi(\boldsymbol{\theta})\right\}}$,

where the normalization by $\max _{\boldsymbol{\theta}}\left\{\pi_{\text {initial }}(\boldsymbol{\theta}), \pi(\boldsymbol{\theta})\right\}$ is included in oder to ensure that the possibility distribution $\pi_{\mathrm{agg}}$ has a maximum empirical value equal to one.

The possibilistic method proposes the use of more than one performance criteria, in order to evaluate different aspects of the model's fitness. The normalized product conjunction rule is repeatedly applied for combining the possibility distribution $\pi_{\text {initial }}$ and the possibility distributions associated with the different measures of model performance being considered. Supossing that a total of $N$ different measures of model performance are used, it can be shown that the overall possibility distribution of the parameter vector is given by

$$
\begin{aligned}
& \pi_{\text {agg }_{-} 1,2, \ldots, N}(\boldsymbol{\theta}) \\
& =\frac{\pi_{\text {initial }}(\boldsymbol{\theta}) \cdot \pi_{1}(\boldsymbol{\theta}) \cdot \pi_{2}(\boldsymbol{\theta}) \cdot \ldots \cdot \pi_{N}(\boldsymbol{\theta})}{\max _{\boldsymbol{\theta}}\left\{\pi_{\text {initial }}(\boldsymbol{\theta}) \cdot \pi_{1}(\boldsymbol{\theta}) \cdot \pi_{2}(\boldsymbol{\theta}) \cdot \ldots \cdot \pi_{N}(\boldsymbol{\theta})\right\}},
\end{aligned}
$$

where $\pi_{1}, \pi_{2}, \ldots, \pi_{N}$ represent the possibility distributions induced by the measures of model performance included in the analysis. The use of additional performance criteria is expected to provide new knowledge about the goodness of the model realizations, thus reducing predictive uncertainty. Due to the associativity of the normalized product conjunction, the order in which the individual possibility distributions $\pi_{1}$, $\pi_{2}, \ldots, \pi_{N}$ are included in the analysis does not affect the overall implied possibility distribution $\pi_{\text {agg_1,2,..,N }}$, as seen in Eq. (13).

It has been pointed out that a conjunctive combination rule is only justifiable if all sources of information are seen as equally reliable (Dubois and Prade, 1994, 1998), as assumed in the paper where the possibilistic method was first presented (Jacquin and Shansledin, 2007) and also in the present study. The normalized product operator is chosen because it allows a reinforcement of possibility degrees and it is also associative, which are advantages with respect to the normalized minimum operator (Dubois and Prade, 1994). It is worth noting that a conjunctive combination rule would be inapplicable if there was total conflict between the sources of information being combined. However, if the model structure is indeed appropriate for modelling the runoff generation process of the catchment, it is unlikely that a total conflict exists between the few measures of model performance that are used for the evaluation of model performance. In this case, it is expected that several parameter vectors can be found that are able to produce estimated discharge hydrographs that approximately fit the observations, obtaining relatively good performance indices with respect to all the measures of model performance being considered. It is possible that adaptive combination rules (see e.g. Dubois and Prade, 1994; Destercke et al., 2009), useful when there is a level of conflict between sources of information, would also provide an appropriate solution in this situation.

\subsection{Derivation of prediction uncertainty bounds}

The possibility distribution of the discharge predictions at each time step $t$ is empirically derived from the aggregate possibility values $\pi_{\mathrm{agg}}(\boldsymbol{\theta})$ of the parameter vectors in the sample. Given a particular model structure and input data, the model output $Q_{t}^{*}$ at time $t$ is a deterministic function of the parameter vector $\boldsymbol{\theta}$. By virtue of the Extension Principle, the possibility distribution of the discharge estimates $Q_{t}^{*}$ at time step $t$ is given by

$$
\pi_{\mathrm{agg}}^{(t)}\left(q^{*}\right)\left\{\begin{array}{c}
\max _{t}^{*}(\boldsymbol{\theta})=q^{*} \\
0, \text { if } Q_{t}^{*}(\boldsymbol{\theta}) \neq q^{*} \text { for all } \boldsymbol{\theta}
\end{array},\right.
$$


where $q^{*}$ is a possible value of $Q_{t}^{*}$. Equation (14) implies that model realizations with possibility values $\pi_{\mathrm{agg}}(\boldsymbol{\theta})$ equal to zero are implicitly discarded from the sample, because only simulations with possibility values $\pi_{\mathrm{agg}}(\boldsymbol{\theta})$ strictly greater than zero do effectively contribute in the derivation of the uncertainty bounds.

The upper and lower bounds of the strong $\alpha$-cuts of the possibility distribution $\pi_{\mathrm{agg}}^{(t)}$, i.e. the set of all values $q^{*}$ with possibility values $\pi_{\mathrm{agg}}^{(t)}\left(q^{*}\right)$ strictly greater than $\alpha$, define the $\alpha$ possibility bounds of the discharge predictions. It is worth discussing the distinction between the $\alpha$ possibility bounds and the strong $\alpha$-cuts, because this may cause some confusion. As explained in Sect. 2.4, all the strong $\alpha$-cuts of $\pi_{\mathrm{agg}}^{(t)}$ are open intervals if the possibility distribution $\pi_{\text {agg }}^{(t)}$ is continuous and unimodal; hence, the interval of discharge estimates enclosed by the $\alpha$ possibility bounds is exactly the same as the strong $\alpha$-cut of $\pi_{\text {agg }}^{(t)}$ in this case. However, if the possibility distribution $\pi_{\text {agg }}^{(t)}$ has several local maxima, the set of discharge estimates enclosed by the $\alpha$ possibility bounds and the discharge estimates inside the strong $\alpha$-cut are not the same at all possibility levels. In this situation, only possibility levels that that are higher than all local maxima different from the global maximum, and possibility levels that are lower than all local minima define strong $\alpha$-cuts that are intervals; other possibility levels define strong $\alpha$-cuts that are given by the union of two or more intervals, implying that the $\alpha$ possibility bounds enclose a range of discharge estimates whose possibility values are not all greater than $\alpha$. In any case, whether or not the range of discharge predictions enclosed by the $\alpha$ possibility bounds coincide with the strong $\alpha$-cuts, Eq. (5) implies that the necessity degree of the interval of discharge predictions inside the $\alpha$ possibility bounds is equal to $1-\alpha$.

\section{Case study}

\subsection{Snowmelt runoff model}

The model analyzed is a conceptual type snowmelt runoff model that is widely used in water resources studies for the mining industry in Chile (e.g. Water Management Ltda., 2001; Arcadis Geotécnica, 2007). The version of the model used here (Kamann, 1998) operates at a monthly time step. The hydrometeorological information required includes precipitation, number of rain days, evaporation, temperature, air humidity, wind speed and cloud cover. The model output is given by the monthly discharge at the catchment's outlet. In the manner computationally implemented in this study, the model has a total of 16 independent parameters.

The model divides the catchment into five elevation zones, where the fifth zone corresponds to the catchment glaciers. Snowmelt is calculated using an energy balance method, where incident solar radiation is estimated with an empirical

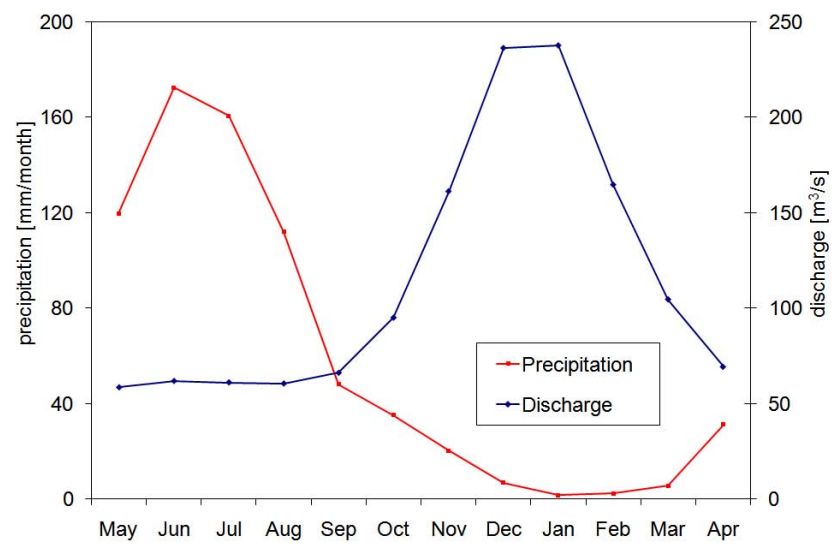

Fig. 2. Seasonal evolution of monthly precipitation and monthly mean discharge in Maipo at El Manzano catchment.

formula locally adjusted for the Andes Mountains of Central Chile (Espíldora, 1968) and albedo values are obtained from empirical curves (Amorocho and Espíldora, 1966). In the case of the fifth elevation zone, glaciers are seen as an inexhaustible source of water that melts when the snow cover is depleted. An individual surface-soil moisture balance is performed within each elevation zone, in order to generate its contribution to direct runoff and groundwater recharge. With the aim of simulating the diffusion and attenuation effects of the catchment, routing elements are incorporated to the model. Direct runoff components from the individual elevation zones are routed through separate linear reservoirs; the catchment's total direct runoff is finally obtained by sumation of the routed direct runoff contributions from the individual elevation zones. Groundwater recharge at the catchment level is calculated as the sum of groundwater recharge from the individual elevation zones and further routed through a single linear reservoir, in order to obtain the total generated groundwater runoff. Total estimated discharge corresponds to the sum of total surface and total groundwater runoff.

\subsection{Catchment and data}

The study area is located in the Andean region of Central Chile. Maipo River at El Manzano is a snow dominated catchment with a surface of $4968\left[\mathrm{Km}^{2}\right]$, where approximately $8 \%$ was covered by glaciers at the time when the data used in this study were collected (Valdivia, 1984). Elevation ranges from 890 [m a.s.1.] to 6570 [m a.s.1.], with a median altitude of 3200 [m a.s.l.]. Glacier areas are located above 3500 [m a.s.1.] (Valdivia, 1984).

Precipitation is mostly produced by cold fronts that arrive in the area during winter. Accordingly, as shown in Fig. 2, most precipitation occurs between May and August, while precipitation amounts during the rest of the year are relatively low. The observed snowline in the area is located about 2100 [m a.s.l.] during May-September, which 
implies that most precipitation corresponds to snowfall. Except for snow and glacier zones in the higher areas, snow cover in the catchment is lost by the end of the melting period. As seen in Fig. 2, monthly mean discharge is minimal in May-August, but it increments during the melting season September-March; monthly discharge reaches its maximum value in December or January. Human intervention in the catchment's hydrological regime at the time when the data used in this study were collected was not significant. Glacier mass balance studies in the area are scarce. However, it has been estimated that glaciers in Central Chile experienced an average mass loss $\Delta h_{\text {med }}=0.45-0.95[\mathrm{~m} /$ year] of equivalent water depth in the period 1945-1996 (Rivera et al., 2002). This mass loss does not occur in a systematic manner, as negative mass balances alternate with positive mass balances in El Niño years.

In this study, the hydrological year is defined from 1 May (coinciding with the minimum monthly mean discharge and also the beginning of heavy precipitation) to 30 April. Data available for the study consists of monthly time series during the hydrological years 1962/19631990/1992. The available data were divided into a calibration period (1962/1963-1982/1983) and a verification period (1983/1984-1990/1991) for split sampling tests. The first year of calibration is used as a warming-up period.

\section{Methodology}

\subsection{Sample of model realizations and initial possibility values}

A sample of the parameter vector is generated by varying all 16 parameters simultaneously and independently. The sample of model parameters is generated by assuming that each parameter has a uniform probability distribution within its feasible range, implying that the parameter vectors in the sample are uniformly distributed in the parameter space. The feasible ranges for the model parameters are defined so that they are wider than the ranges of optimal parameter values found in previous applications of the model in other catchments. Preliminary experiments with varying sample sizes were performed, with the aim of establishing what sample size is appropriate for the model and the catchment case study. The chosen sample size is 80,000 , because it was observed that further increases in the number of parameter vectors in the sample does not produce significant changes in the possibility distributions of discharge estimates.

Initial possibility values of the parameter vectors in the sample are calculated according to

$\pi_{\text {initial }}(\boldsymbol{\theta})=\left\{\begin{array}{l}1, \text { if } \boldsymbol{\theta} \in \Omega \\ 0, \text { otherwise }\end{array}\right.$,

where $\Omega$ is the feasible space of the parameter vector. Equation (15) implies that the initial possibility values $\pi_{\text {initial }}(\boldsymbol{\theta})$ of all the parameter vectors in the sample, whose elements are necessarily inside the corresponding feasible ranges, are assigned an initial possibility value of unity. This definition is consistent with the fact that, even though there is prior knowledge on the ranges where the optimal parameter values are usually found when the model is calibrated, a lot of dispersion exists between catchments. Accordingly, it is not known a priori what regions of the parameter space are more likely to be associated with good model performance in the catchment case study.

\subsection{Evaluation of model performance}

The possibility distributions of the parameter vector are subsequently obtained through evaluation of the goodness of fit of the estimated discharge hydrographs. Model performance is first evaluated according to the mean squared error of the Box-Cox transformed discharge $\left(\mathrm{MSE}_{\mathrm{BC}}\right)$ as seen in previous studies (Thiemann et al., 2001; Misirli et al., 2003), which reduces the effect of heteroscedasticity and emphasizes the importance of the model performance during low flow periods. The associated possibility distribution is defined by

$\pi_{1}(\boldsymbol{\theta})=\left\{\begin{array}{l}\frac{V_{\mathrm{BC}}-\operatorname{MSE}_{\mathrm{BC}}(\boldsymbol{\theta})}{V_{\mathrm{BC}}-\min _{\boldsymbol{\theta}}\left\{\mathrm{MSE}_{\mathrm{BC}}(\boldsymbol{\theta})\right\}}, \operatorname{MSE}_{\mathrm{BC}}(\boldsymbol{\theta}) \leq V_{\mathrm{BC}} \\ 0, \text { otherwise }\end{array}\right.$,

where $V_{\mathrm{BC}}$ is the variance of the Box-Cox transformed observed discharge during the calibration period, and $\min _{\boldsymbol{\theta}}\left\{\operatorname{MSE}_{\mathrm{BC}}(\boldsymbol{\theta})\right\}$ represents the lowest $\operatorname{MSE}_{\mathrm{BC}}(\theta)$ value found among all the model realizations in the sample. The chosen model rejection criterion specifies that model realizations with $\operatorname{MSE}_{\mathrm{BC}}(\boldsymbol{\theta})$ values greater than $V_{\mathrm{BC}}$ are assigned possibility values equal to zero. The choice of this behavioural threshold is based on the interpretation that a $\operatorname{MSE}_{\mathrm{BC}}(\boldsymbol{\theta})$ value greater than $V_{\mathrm{BC}}$ indicates that the model is outperformed by a naïve model whose Box-Cox transformed output is always equal to the mean Box-Cox transformed observed discharge during the calibration period.

The second possibility distribution used for constraining the model representations is based on the volumetric error (Jacquin and Shamseldin, 2007). This possibility distribution is defined as

$\pi_{2}(\boldsymbol{\theta})=\left\{\begin{array}{l}\frac{1-|\operatorname{REVF}(\boldsymbol{\theta})|}{1-\min _{\boldsymbol{\theta}}\{|\operatorname{REVF}(\boldsymbol{\theta})|\}}, 0 \leq|\operatorname{REVF}(\boldsymbol{\theta})| \leq 1 \\ 0, \text { otherwise }\end{array}\right.$,

where $\operatorname{REVF}(\boldsymbol{\theta})$ represents the relative error of the volumetric fit of the model realization, given by

$\operatorname{REVF}(\boldsymbol{\theta})=1-\frac{\sum Q_{t}^{*}(\boldsymbol{\theta})}{\sum Q_{t}}$.

The quantity $\min _{\boldsymbol{\theta}}\{|\operatorname{REVF}(\boldsymbol{\theta})|\}$ corresponds to the smallest $|\operatorname{REVF}(\boldsymbol{\theta})|$ value found in the sample of model realizations. The model rejection criterion implicit in Eq. (17) consists in 
the removal of the model realizations with absolute volumetric errors greater than $100 \%$ during the calibration period.

The last possibility distribution used in this study is intended to asses the ability of the models to estimate the discharge peaks. The value of this possibility distribution is calculated according to

$\pi_{3}(\boldsymbol{\theta})=\left\{\begin{array}{l}\frac{1-\operatorname{REP}(\boldsymbol{\theta})}{1-\min _{\boldsymbol{\theta}}\{\operatorname{REP}(\boldsymbol{\theta})\}}, 0 \leq \operatorname{REP}(\boldsymbol{\theta}) \leq 1 \\ 0, \text { otherwise }\end{array}\right.$,

where REP $(\boldsymbol{\theta})$ represents the average relative error to the peak of the model realization. This statistic is given by

$\operatorname{REP}(\boldsymbol{\theta})=\sum_{i=1}^{N_{\mathrm{p}}} \frac{\left|Q p_{t}-Q p_{t}^{*}(\boldsymbol{\theta})\right|}{N_{\mathrm{p}} Q p_{t}}$,

where $N_{\mathrm{p}}$ is the number of selected flow peaks, $Q p_{t}$ represents a peak in the observed hydrograph, and $Q p_{t}^{*}(\boldsymbol{\theta})$ is the model estimated discharge for the same time step as $Q p_{t}$. The quantity $\min _{\boldsymbol{\theta}}\{\operatorname{REP}(\boldsymbol{\theta})\}$ in Eq. (19) represents the smallest REP $(\boldsymbol{\theta})$ value among all the model realizations in the sample. The model rejection criterion specified by Eq. (19) consists in the removal of the realizations whose REP $(\boldsymbol{\theta})$ values are greater than $100 \%$ during the calibration period.

The normalization factors $V_{\mathrm{BC}}-\min _{\boldsymbol{\theta}}\left\{\operatorname{MSE}_{\mathrm{BC}}(\boldsymbol{\theta})\right\}$, $1-\min _{\boldsymbol{\theta}}\{|\operatorname{REVF}(\boldsymbol{\theta})|\}$ and $1-\min _{\boldsymbol{\theta}}\{\operatorname{REP}(\boldsymbol{\theta})\}$ in Eqs. (16), (17) and (19), respectively, are introduced in order to obtain possibility values with a maximum empirical value of unity. The rationale of this choice is that the simulation providing the most plausible representation of the real system, as indicated by the measure of model performance used in each case, is assigned a possibility value equal to unity. Model rejection criteria more restrictive than those specified by Eqs. (16), (17) and (19) are not considered necessary within the possibilistic framework, as model realizations with low possibility values do not affect the uncertainty bounds at high possibility levels.

The possibility distribution $\pi_{1}$ gives an indication of the goodness of fit of the estimated discharge hydrograph in all flow ranges. For this reason, this possibility distribution is seen as a primary source of information on model performance in this study. The possibility distribution $\pi_{2}$ evaluates the average error of the discharge predictions, but it does not indicate how close the estimated discharge hydrograph is to the individual observations. Similarly, the possibility distribution $\pi_{3}$ highlights the goodness of the model estimated discharge peaks, but it does not provide information on the performance of the model in other flow ranges. Therefore, the possibility distributions $\pi_{2}$ and $\pi_{3}$ are not used alone for constraining the model representations. In this study, the information supplied by these possibility distributions is used as a complement to that provided by the possibiliy distribution $\pi_{1}$.

\subsection{Aggregate possibility distributions of the parameter vector}

Once the possibility values $\pi_{\text {initial }}(\boldsymbol{\theta}), \pi_{1}(\boldsymbol{\theta}), \pi_{2}(\boldsymbol{\theta})$ and $\pi_{3}(\boldsymbol{\theta})$ have been obtained, aggregate possibility values are derived using the combination rule of Eq. (13). The first aggregate possibility distribution defined, $\pi_{\text {agg_1 }}$, uses only the information provided by the mean squared error of the BoxCox transformed discharge for constraining the model representations. Accordingly, the possibility values $\pi_{\mathrm{agg}_{-1}}(\boldsymbol{\theta})$ are obtained after substitution of the values $\pi_{\text {initial }}(\boldsymbol{\theta})$ and the values $\pi_{1}(\boldsymbol{\theta})$ in Eq. (13). The aggregate possibility distribution $\pi_{\text {agg_1,2 }}$ further includes the information on the volumetric fit of the model realizations provided by the possibility distribution $\pi_{2}$. The possibility values $\pi_{\text {agg_1,2 }}(\boldsymbol{\theta})$ are thus obtained by substituting $\pi_{\text {initial }}(\boldsymbol{\theta}), \pi_{1}(\boldsymbol{\theta})$ and $\pi_{2}(\boldsymbol{\theta})$ in Eq. (13). Finally, the information on the ability of the models to estimate the discharge peaks, provided by the possibility distribution $\pi_{3}$, is used for further constraining the model representations. Hence, the values of the aggregate possibility distribution $\pi_{\text {agg_1 } 1,2,3}$ are calculated by substituting $\pi_{\text {initial }}(\boldsymbol{\theta}), \pi_{1}(\boldsymbol{\theta}), \pi_{2}(\boldsymbol{\theta})$ and $\pi_{3}(\boldsymbol{\theta})$ in Eq. (13).

\subsection{Derivation of prediction uncertainty bounds}

As explained in Sect. 3.4, the possibility distribution of the discharge predictions is empirically derived from the information provided by the aggregate possibility values of the parameter vectors in the sample. The possibility values $\pi_{\text {agg_1 }}(\boldsymbol{\theta}), \pi_{\text {agg_1,2 }}(\boldsymbol{\theta})$ and $\pi_{\text {agg_1,2,3 }}(\boldsymbol{\theta})$ are substituted in Eq. (14) for obtaining the possibility values $\pi_{\text {agg_1 }_{1}}^{(t)}\left(q^{*}\right)$, $\pi_{\text {agg_1,2 }}^{(t)}\left(q^{*}\right)$ and $\pi_{\text {agg_1,2,3 }}^{(t)}\left(q^{*}\right)$, respectively. Possibility bounds of these possibility distributions are derived at several possibility levels $\alpha$, in order to evaluate the effect of the possibility level on the characteristics of the uncertainty bounds.

\subsection{Alternative definition of the initial possibility distribution}

An alternative definition of the initial possibility distribution of the model parameters is also tested. This initial possibility distribution, which evaluates the likelihood of the simulated glacier mass balance and snow cover at the end of the calibration period, is given by

$\pi_{0}(\boldsymbol{\theta})$

$=\left\{\begin{array}{l}1, \text { if }(\boldsymbol{\theta} \in \Omega) \text { and }(\text { glacbal }>-2[\mathrm{~m} / \text { year }] \cdot N \mathrm{cal}) \text { and }(\text { snowac }=0) \\ 0, \text { otherwise. }\end{array}\right.$

The variable glacbal in Eq. (21) represents the accumulated surface mass balance between precipitation, evapotranspiration and melt in the glacier zone, from the end of the warming-up period until the end of the calibration period. $N$ cal is the number of years of the calibration period. Accumulated glacier mass losses exceeding 2 times the average values reported in the literature for the study area (see 
Sect. 4.2) are considered unrealistic and the possibility values $\pi_{0}(\boldsymbol{\theta})$ of these model realizations are set to zero. The variable snowac in Eq. (21) represents the snow water equivalent accumulated in the elevations zones below the $2{ }^{\circ} \mathrm{C}$ isothermal line by the end of the calibration period, which should be null according to what was discussed in Sect. 4.2. Model realizations yielding snow accumulations that do not fulfill this requirement are also assigned possibility values $\pi_{0}(\boldsymbol{\theta})$ equal to zero.

In analogy to what was described in Sect. 5.3, aggregate possibility distributions of the parameter vector are defined using $\pi_{0}$ as initial possibility distribution instead of $\pi_{\text {initial }}$. The aggregate possibility distributions thus defined are denoted $\pi_{\text {agg_0 } 0,1}, \pi_{\text {agg_0 } 0,1,2}$ and $\pi_{\text {agg_0 } 0,1,2,3}$. Finally, the aggregate possibility values $\pi_{\text {agg_o, }}(\boldsymbol{\theta}), \pi_{\text {agg_0,1,2 }}(\boldsymbol{\theta})$ and $\pi_{\text {agg_0 } 0,1,2,3}(\theta)$ are substituted in Eq. (14) for empirically deriving possibility distributions of the discharge estimates (named $\pi_{\text {agg } 0,1}^{(t)}, \pi_{\text {agg }_{0} 0,1,2}^{(t)}$ and $\pi_{\text {agg } 0,1,2,3}^{(t)}$, respectively) and prediction uncertainty bounds.

\section{Results}

\subsection{Number of simulations retained according to different criteria}

Firstly, it was observed that the effective sample size notably reduces when the possibility distribution $\pi_{0}$ is used as initial possibility distribution of the parameter vector. In particular, only 28859 simulations among 80000 in the sample obtained initial possibility values $\pi_{0}(\boldsymbol{\theta})$ greater than zero. This situation is mainly due to the restriction imposed on the simulated snow cover, which was only fulfilled by 28902 model realizations. By contrast, the restriction imposed on the mass balance in the glaciers was achieved by most of the simulations (75 043).

Figure 3 shows the total number of simulations retained above different possibility levels $\alpha$ of the possibility distributions $\pi_{\text {agg_1 }}, \pi_{\text {agg_1,2 }}$ and $\pi_{\text {agg_1,2,3 }}$. This figure reveals that the rejection criterion specified by the possibility distribution $\pi_{1}$ is quite restrictive, as the number of simulations having possibility values $\pi_{\text {agg_1 }}(\boldsymbol{\theta})$ greater than zero is about $40 \%$ the total number of model realizations in the sample. Figure 3 also demonstrates that the total number of simulations retained above a given possibility level $\alpha$ decreases as more information is used for defining the aggregate possibility distribution of the model representations. The most notable reductions are seen when the information on the peak errors is included in the definition of the aggregate possibility distribution of the parameter vector (i.e. when using $\pi_{\text {agg_1,2,3 }}$ instead of $\pi_{\text {agg_1,2 }}$ ). The conclusions drawn from the analysis of the number of simulations retained above different possibility levels of the possibility distributions $\pi_{\text {agg_0,1 }}, \pi_{\text {agg_0,1,2 }}$ and $\pi_{\text {agg_0,1,2,3 }}$ are analogous to those discused with respect to $\pi_{\text {agg_1 }}, \pi_{\text {agg_1 } 1,2}$ and $\pi_{\text {agg_1,2,3 }}$.

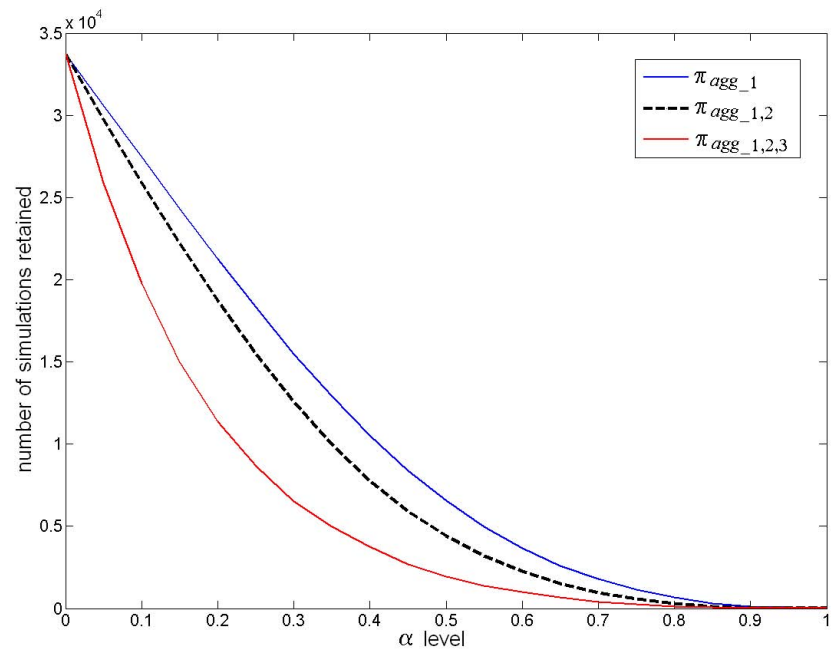

Fig. 3. Total number of simulations retained above different possibility levels $\alpha$ of the possibility distributions $\pi_{a g g_{-} 1}, \pi_{\text {agg_1,2 }}$ and $\pi_{\text {agg_1,2,3. }}$.

\subsection{Performance of the simulations retained according to different criteria}

Figure 4 shows ranges of model efficiency $R^{2}$ (Nash and Sutcliffe, 1970), REVF and REP values obtained during the verification period by the simulations retained above different possibility levels $\alpha$ of the possibility distributions $\pi_{\text {agg_1 } 1}, \pi_{\text {agg_1 } 1,2}$ and $\pi_{\text {agg_1 } 1,2,3}$. Figure 4 demonstrates that $R^{2}, \mid$ REVF $\mid$ and REP values of some of the simulations retained about the possibility level $\alpha=0$ are quite poor (i.e. low $R^{2}$ values, high $|\mathrm{REVF}|$ and high REP values). Including more information in the model selection criterion does not help to remove these underperforming simulations, unless the possibility level $\alpha$ is increased. At possibility levels $\alpha>0$, the lower bound of the efficiency values $R^{2}$ observed during the verification period can normally be raised by moving from the possibility distribution $\pi_{\text {agg_1 }}$ to $\pi_{\text {agg_1,2 }}$; a further increase in this lower bound is normally achieved if $\pi_{\text {agg_1 } 1,3,3}$ is used instead of $\pi_{\text {agg_1 } 1,2}$. Similarly, replacing the possibility distribution $\pi_{\text {agg_1 } 1}$ by $\pi_{\text {agg_1,2 }}$ usually produces a significant decrease in the highest $|\mathrm{REVF}|$ and REP values observed during the verification period at possibility levels $\alpha>0$; in general, a further improvement in $|\mathrm{REVF}|$ and REP values is observed if $\pi_{\text {agg_1,2,3 }}$ is used instead of $\pi_{\text {agg_1,2 }}$.

Figure 5 shows ranges of model efficiency $R^{2}$, REVF and REP values obtained during the verification period by the simulations retained above different possibility levels $\alpha$ of the possibility distributions $\pi_{\text {agg_0,1 }}, \pi_{\text {agg_0,1,2 }}$ and $\pi_{\text {agg_0 } 0,1,2,3}$. Analysis of Fig. 5 reveals that these results are analogous to those observed in the case of the possibility distributions $\pi_{\text {agg_1 }}, \pi_{\text {agg_1,2 }}$ and $\pi_{\text {agg_1,2,3 }}$. In particular, including more information in the definition of the possibility distribution of the model realizations does not help to remove 

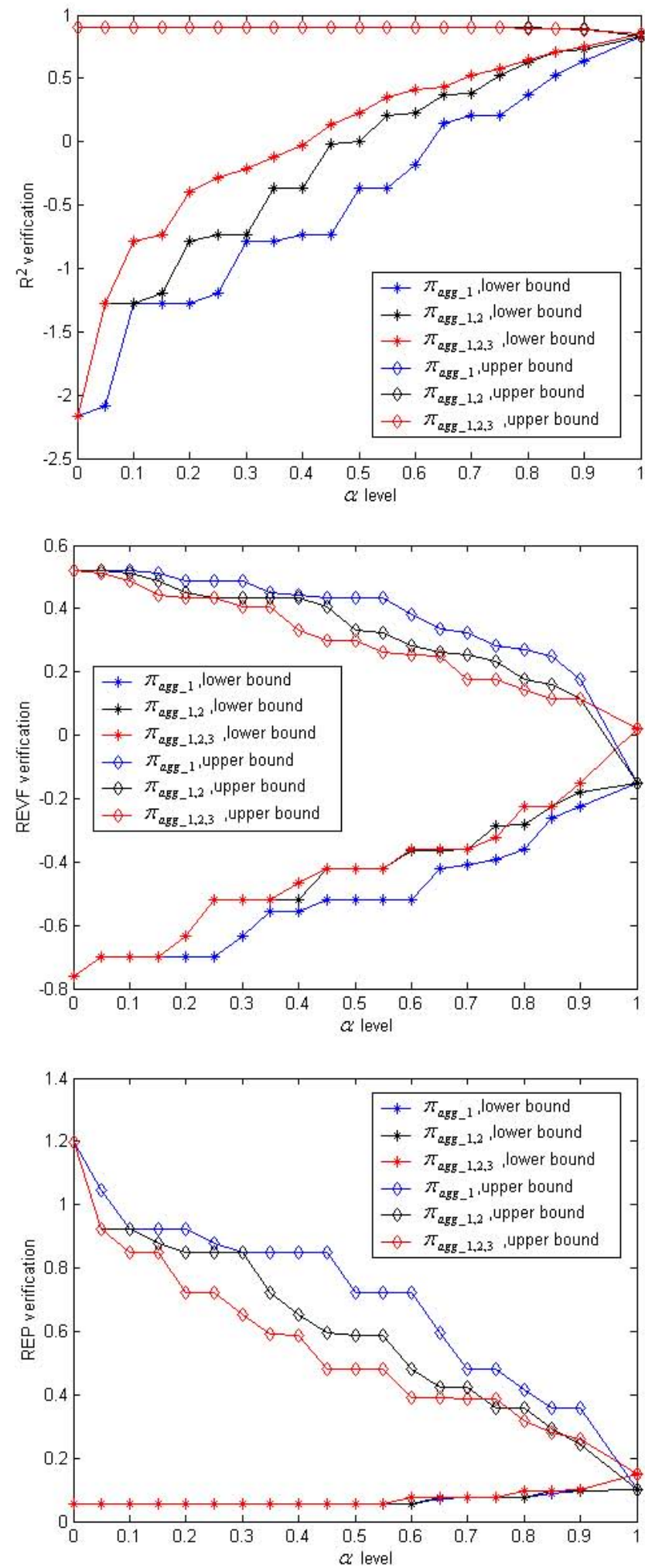

Fig. 4. Ranges of model performance statistics during the verification period of the simulations retained above given possibility levels $\alpha$ of the possibility distributions $\pi_{\text {agg_1 }}, \pi_{\text {agg_1,2 }}$ and $\pi_{\text {agg_1,2,3 }}$.
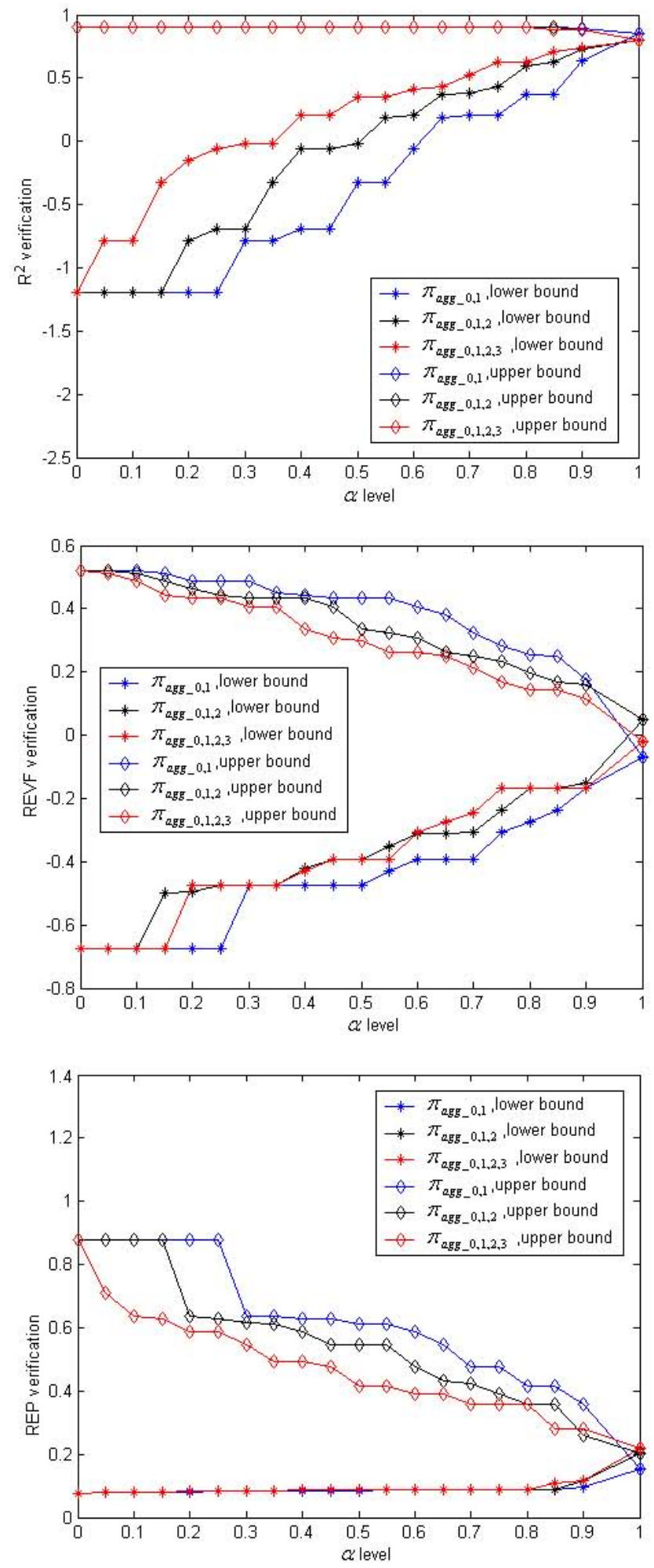

Fig. 5. Ranges of model performance statistics during the verification period of the simulations retained above given possibility levels $\alpha$ of the possibility distributions $\pi_{\text {agg_0,1 }}, \pi_{\text {agg_0,1,2 }}$ and $\pi_{\text {agg_0, } 1,2,3}$. 


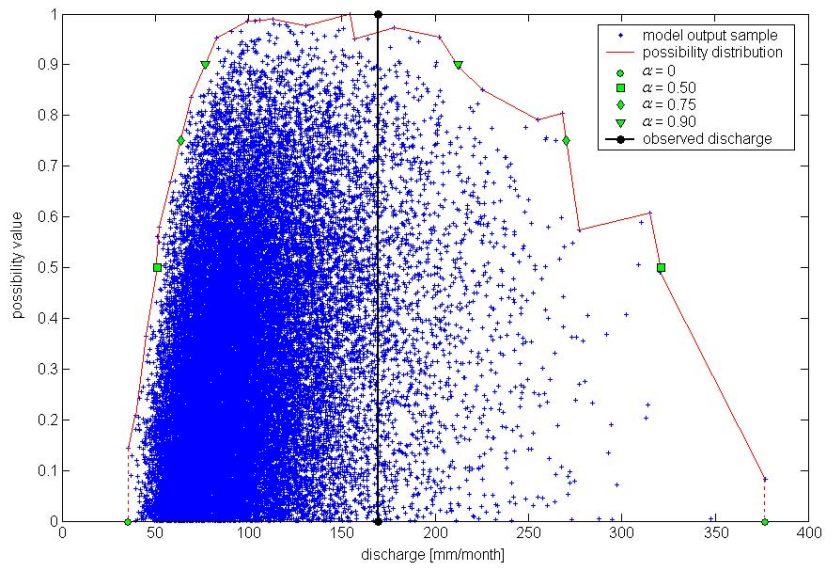

Fig. 6. Possibility distribution of the discharge estimates on December 1983 empirically derived from the possibility values $\pi_{\text {agg_1 }}(\boldsymbol{\theta})$, observed discharge and selected possibility bounds.

underperforming simulations at the possibility level $\alpha=0$. However, the performance of the simulations retained above possibility levels $\alpha>0$ generally improves when moving from $\pi_{\text {agg_0 } 0,1}$ to $\pi_{\text {agg_0 } 0,1,2}$, and from $\pi_{\text {agg_0 } 0,1,2}$ to $\pi_{\text {agg_0 } 0,1,2,3}$. Comparison of Figs. 4 and 5 further reveals that using the initial possibility distribution $\pi_{0}$ instead of $\pi_{\text {initial }}$ results in an improvement in the performance of the simulations retained at low-medium possibility levels, although this positive feature is not so evident at high possibility levels.

\subsection{Possibility bounds of the discharge estimates}

Figure 6 shows an example of the empirical derivation of the possibility distribution of the discharge estimates. In particular, Fig. 6 shows the possibility distribution of the discharge estimates on December 1983 derived from the possibility values $\pi_{\text {agg_1 }}(\boldsymbol{\theta})$; the observed monthly discharge and the uncertainty bounds at the possibility levels $\alpha=0, \alpha=0.50$, $\alpha=0.75$ and $\alpha=0.90$ are also indicated for reference. Each cross in this plot represents the combination of a model estimated discharge $Q_{t}^{*}\left(\boldsymbol{\theta}^{i}\right)$ and the corresponding possibility value $\pi_{\text {agg_1 }}\left(\boldsymbol{\theta}^{i}\right)$, where $\boldsymbol{\theta}^{i}$ represents the parameter vector with which that particular discharge estimate was obtained. The empirical possibility distribution $\pi_{\text {agg_1 }}^{(t)}$ is given by the envelope of these sample points, obtained by partitioning the range of discharge estimates (at the corresponding time step) into several intervals. As reported in previous applications of the method (Jacquin and Shamseldin, 2007, 2009), the empirical possibility distributions of the discharge estimates have minor oscillations, i.e. they have several local maxima (see Fig. 6). Although this feature may be due to the fact that the envelopes are obtained from a sample of finite size, it is also possible that this is an intrinsic characteristic of the possibility distributions. Hence, it is not guaranteed that the possibility bounds at all possibility levels $\alpha$ enclose
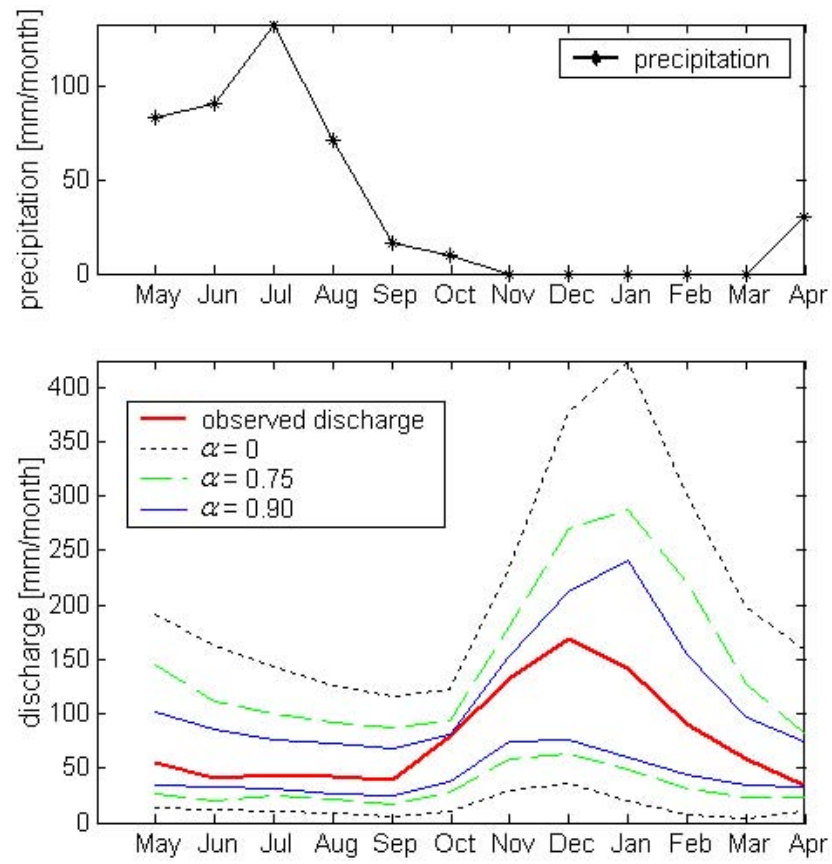

Fig. 7. Precipitation history, observed discharge and selected possibility bounds derived from the aggregate possibility distribution $\pi_{\text {agg_1 }}$ for the hydrological year $1983 / 1984$.

only discharge estimates with possibility values greater than $\alpha$, as discussed earlier in Sect. 3.4. The 0 possibility bounds in Fig. 6 are obtained by including the discharge estimates from of all the simulations with possibility values $\pi_{\mathrm{agg}_{-1}}(\boldsymbol{\theta})$ strictly greater than zero, that is, not rejected according to the criterion specified by Eq. (16). Hence, the interval of discharge predictions within these possibility bounds has a necessity degree of unity, as explained in Sect. 3.4. Similarly, the necessity degrees of the intervals of discharge predictions inside the uncertainty bounds at the possibility levels $\alpha=0.50, \alpha=0.75$ and $\alpha=0.90$ are $0.50,0.25$ and 0.10 , respectively.

Figure 7 shows selected possibility bounds $(\alpha=0, \alpha=0.75$ and $\alpha=0.90$ ) for the hydrological year 1983/1984; this figure corresponds to the case where the aggregate possibility distribution of the parameter vector is given by $\pi_{\text {agg_1 }}$. The concurrent time series of rainfall amounts and observed discharges are also shown. Similarly, Fig. 8 shows selected possibility bounds derived from the aggregate possibility distribution $\pi_{a g g_{-} 1,2,3}$ for the hydrological year $1983 / 1984$. Not surprisingly, Figs. 7 and 8 demonstrate that increasing the possibility level $\alpha$ reduces the width of the prediction intervals within the possibility bounds (see also Fig. 6). More interestingly, it can be observed that the uncertainty in the predictions of the model is generally large with respect to the magnitude of the concurrent discharge observations. Moreover, the distance between the uncertainty bounds tends to increase with the magnitude of the observed discharge, 


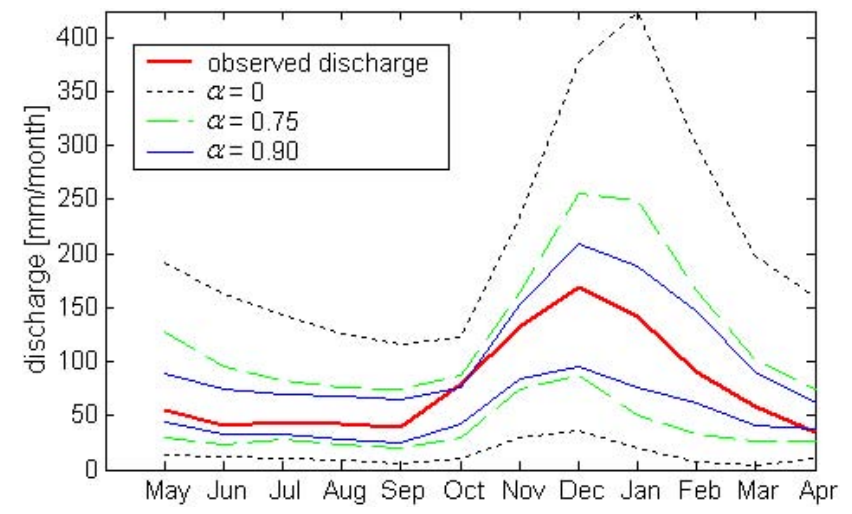

Fig. 8. Observed discharge and selected possibility bounds derived from the aggregate possibility distribution $\pi_{\text {agg_1,2,3 }}$ for the hydrological year 1983/1984.

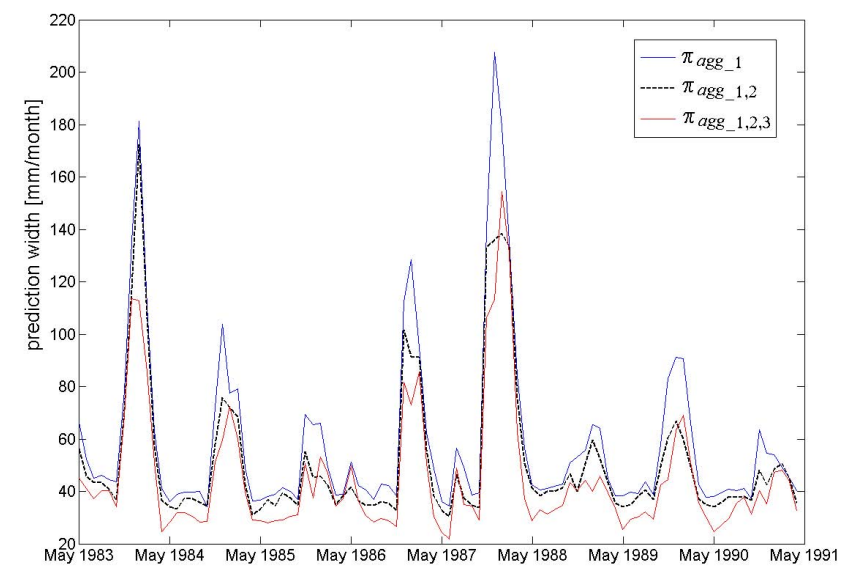

Fig. 9. Prediction width at the possibility level $\alpha=0.90$ for the aggregate possibility distributions $\pi_{\text {agg_1 }}, \pi_{\text {agg_1,2 }}$ and $\pi_{\text {agg_1,2,3 }}$ during the verification period.

which indicates an increase in predictive uncertainty. However, incorporating more information in the calculation of the aggregate possibility distribution of the parameter vector generally has a narrowing effect in the possibility bounds, which reduces predictive uncertainty. For example, Fig. 9 shows the prediction width at the possibility level $\alpha=0.90$ for the aggregate possibility distributions $\pi_{\text {agg_1 } 1}, \pi_{\text {agg_1,2 }}$ and $\pi_{\text {agg_1,2,3 }}$. Similarly, a reduction of prediction width generally occurs if the definition of the initial possibility distribution changes from $\pi_{\text {initial }}$ to $\pi_{0}$, as seen in Fig. 10 .

As seen in previous studies (e.g. Montanari, 2005; Jacquin and Shamseldin, 2007), the performance of the uncertainty bounds is assessed in terms of their ability to enclose the discharge observations. Table 1 shows the fraction of the observations outside selected possibility bounds $(\alpha=0, \alpha=0.75$ and $\alpha=0.90$ ) of different aggregate possibility distributions during the verification period. As discussed above, incorporating more information in the calculation of the aggregate
Table 1. Fraction of observations not enclosed by the possibility bounds at selected possibility levels $\alpha$ during the verification period.

\begin{tabular}{llccc}
\hline Initial possibility & Aggregate possibility & \multicolumn{4}{c}{$\alpha$ level } \\
\cline { 3 - 5 } distribution & distribution & 0 & 0.75 & 0.90 \\
\hline \multirow{2}{*}{$\pi_{\text {initial }}$} & $\pi_{\text {agg_1 }}$ & 0.00 & 0.02 & 0.07 \\
& $\pi_{\text {agg_1,2 }}$ & 0.01 & 0.02 & 0.13 \\
$\pi_{0}$ & $\pi_{\text {agg_1,2,3 }}$ & 0.02 & 0.03 & 0.14 \\
& $\pi_{\text {agg_0,1 }}$ & 0.01 & 0.02 & 0.10 \\
& $\pi_{\text {agg_0,1,2 }}$ & 0.01 & 0.02 & 0.11 \\
& $\pi_{\text {agg_0,1,2,3 }}$ & 0.01 & 0.04 & 0.15 \\
\hline
\end{tabular}

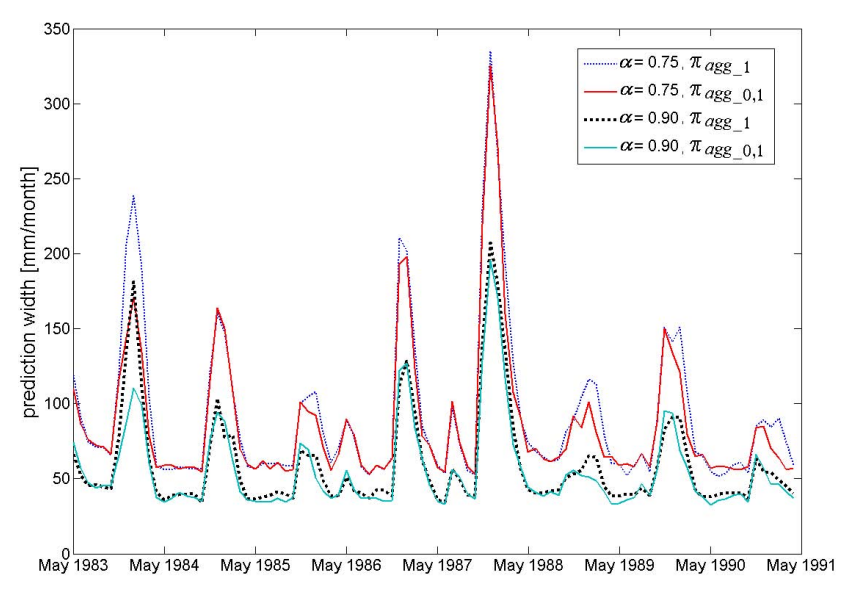

Fig. 10. Prediction width at the possibility levels $\alpha=0.75$ and $\alpha=0.90$ for the aggregate possibility distributions $\pi_{\text {agg_1 }}$ and $\pi_{\text {agg_0,1 }}$ during the verification period.

possibility distribution of the parameter vector has a narrowing effect in the width of the possibility bounds. Consequently, the number of observations not bracketed by the possibility bounds generally increases. This situation is also observed when comparing the fraction of outliers obtained with the initial possibility distribution $\pi_{0}$ and that obtained with the $\pi_{\text {initial }}$, which are generally slightly lower. Table 1 further reveals that the possibility bounds at the possibility levels $\alpha=0$ and $\alpha=0.75$ enclose the majority of the observations; the effect of increasing the possibility level to $\alpha=0.90$ is that the possibility bounds fail to enclose a larger fraction of the observations, although this situation is still unfrequent.

\section{Conclusions}

This study has presented the application of a recently proposed method (Jacquin and Shanseldin, 2007) to the analysis of predictive uncertainty of a conceptual type snowmelt runoff model. This method uses possibilistic rather than probabilistic calculus for the evaluation of predictive uncertainty in watershed modelling. A snow dominated catchment 
in the Chilean Andes is used as case study. Predictive uncertainty arising from parameter uncertainties of the watershed model is assessed using the possibilistic method. The main conclusions of the study are summarized as follows.

It was observed that the number of behavioral simulations (i.e. the model realizations with possibility values $\alpha$ strictly greater than zero) was relatively low compared to the total sample size. This result is in agreement with previous applications of the method (Jacquin and Shamseldin, 2007, 2009). In the case of this study, this situation is mainly due to the severity of the rejection criterion implicit in possibility distribution $\pi_{1}$ based on the mean squared error of the Box-Cox transformed discharge (Eq. 16). The use of the alternative initial possibility distribution $\pi_{0}$, which evaluates the plausibility of the simulated glacier mass balance and snow cover according to Eq. (16), notably reduces the sample size of behavioural simulations. In spite of this filtering process, it was found that the performance of some of the model realizations retained above the posibility level $\alpha=0$ was poor and that using additional model performance criteria did not help to remove these unperforming simulations. At possibility levels $\alpha>0$, however, the performance of the simulations retained tends to improve as more information is used for constraining the model simulations. In particular, using the initial possibility distribution $\pi_{0}$ instead of $\pi_{\text {initial }}$ helps to remove the worst simulations retained at low and medium possibility levels.

In addition to this, it was found that predictive uncertainty of the model is relatively large with respect to the magnitude of the concurrent discharge observations, but using additional information for constraining the model representations allows to reduce it. In particular, a reduction of prediction width is generally achieved if the definition of the initial possibility distribution of the parameter vector assesses the simulated snow cover and glacier mass balance (i.e. if $\pi_{0}$ is used as initial possibility distribution), without a significant increase in the number of observations not enclosed by the possibility bounds. As expected, it was verified that the width of the prediction intervals within the possibility bounds reduces as the possibility level $\alpha$ increases. More importantly, it was also observed that the observed hydrograph was enclosed by the possibility bounds at the possibility levels $\alpha=0$ and $\alpha=0.75$, except in a few cases. Increasing the possibility level to $\alpha=0.90$ reduces the range of predictions retained, at the cost of slightly increasing the fraction of the observations not enclosed by the possibility bounds.

Further research should explore the applicability of other criteria for evaluating model plausibility. At least some of these criteria should constrain the value of the internal variables of the model, in addition to glacier mass balance and snow cover, evaluating the likeliness of the simulated internal processes. This could help to further reduce predictive uncertainty and allow better enclosing the observed hydrographs, without significantly increasing the fraction of outliers.
Acknowledgements. This research was funded by FONDECYT, Research Project 11070130. The author would like to convey her appreciation to the Editor Niko Verhoest and the anonymous referees for their constructive criticisms, which helped to improve the quality of the manuscript.

Edited by: N. Verhoest

\section{References}

Amorocho, J. and Espildora, B.: Mathematical Simulation of the Snow Melting Processes, Water Science and Engineering Paper No. 3001, University of California, Davis, 1966.

Arcadis Geotécnica: Ingeniería Conceptual Solución Ambiental ARD Proyecto Nueva Andina, CODELCO, Chile, 2007.

Arnold, S., Attinger, S., Frank, K., and Hildebrandt, A.: Uncertainty in parameterisation and model structure affect simulation results in coupled ecohydrological models, Hydrol. Earth Syst. Sci., 13, 1789-1807, doi:10.5194/hess-13-1789-2009, 2009.

Ayyub, B. M. and Klir, G. J.: Uncertainty modelling and analysis in engineering and the sciences, Chapman \& Hall, Boca Ratón, 2006.

Bárdossy, A., Mascellani, G., and Franchini, M.: Fuzzy unit hydrograph, Water Resour. Res., 42, W02401, doi:10.1029/2004WR003751, 2006.

Baudrit, C. and Dubois, D.: Comparing methods for joint objective and subjective uncertainty propagation with an example in a risk assessment, $4^{\text {th }}$ International Symposium on Imprecise Probabilities and Their Applications, Pittsburgh, Pensilvania, 31-40, 2005.

Baudrit, C. and Dubois, D.: Practical Representations of Incomplete Probabilistic Knowledge, Comput. Stat. Data An., 51(1), 86-108, 2006.

Bates, B. C. and Townley, L. R.: Nonlinear, discrete flood event models, 3, Analysis of prediction uncertainty, J. Hydrol., 99, 91101, 1988.

Beven, K. J.: A manifesto for the equifinality thesis, J. Hydrol., 320, 18-36, 2006.

Beven, K. and Binley, A.: The future of distributed models: Model calibration and uncertainty prediction, Hydrol. Process., 6, 279298, 1992.

Beven, K. J. and Freer, J.: Equifinality, data assimilation, and uncertainty estimation in mechanistic modelling of complex environmental systems using the GLUE methodology, J. Hydrol., 249, 11-29, 2001.

Cheng, D. K.: Analysis of Linear Systems, Addison-Wesley Publishing Company, Reading, 1959.

Civanlar, M. R. and Trussell, H. J.: Constructing Membership Functions Using Statistical Data, Fuzzy Set. Syst., 18, 1-13, 1986.

Dempster, A. P.: Upper and lower probabilities induced by a multivalued mapping, Ann. Math. Stat., 38(2), 325-339, 1967.

Destercke, S. and Dubois, D.: Possibilistic Information Fusion Using Maximal Coherent Subsets, IEEE T. Fuzzy Syst., 17(1), 7992, 2009.

Dou, C., Woldt, W., Dahab, M., and Bogardi, I.: Transient groundwater flow simulation using a fuzzy set approach, Ground Water, 35(2), 205-215, 1997.

Dubois, D. and Prade, H.: Fuzzy sets and statistical data, Eur. J. Oper. Res., 25, 345-356, 1986. 
Dubois, D. and Prade, H.: When upper probabilities are possibility measures, Fuzzy Set. Syst., 49, 65-74, 1992.

Dubois, D. and Prade, H.: Fuzzy sets and probability: Misunderstandings, bridges and gaps, in: Proceedings 2nd IEEE International Conference on Fuzzy Systems, FUZZ-IEEE'93, San Francisco, California, 28 March-4 April 1993, 1059-1068, 1993.

Dubois, D. and Prade, H.: Possibility theory and data fusion in poorly informed environments, Control Eng. Pract., 2(5), 811823, 1994.

Dubois, D. and Prade, H.: Possibility theory: Qualitative and quantitative aspects, in: Handbook of Defeasible Reasoning and Uncertainty Management Systems, Vol. 1: Quantified Representation of Uncertainty and Imprecision, edited by: Gabbay, D. M. and Smets, P., Kluwer Academic Publishers, Dordrecht, 169226, 1998.

Dubois, D., Foulloy, L., Mauris, G., and Prade, H.: Probabilitypossibility transformations, triangular fuzzy sets, and probabilistic inequalities, Reliab. Comput., 10, 2004, 273-297, 2004.

Dubois, D., Moral, S., and Prade, H.: A semantics for possibility theory based on likelihoods, J. Math. Annal. Appl., 205, 359380, 1997.

Dubois, D., Prade, H., and Smets, P.: A definition of subjective possibility, Int. J. Approx. Reason., 48, 352-364, 2008.

Engeland, K., Xu, C.-Y., and Gottschalk, L.: Assessing uncertainties in a conceptual water balance model using Bayesian methodology, Hydrolog. Sci. J., 50(1), 45-63, 2005.

Espíldora, B.: Instalación y operación de un laboratorio de hidrología de nieves, Seccion Hidrologia y Riego, Departamento de Obras Civiles, Universidad de Chile, Chile, 1968.

Ferson, S. and Ginzburg, L. R.: Different methods are needed to propagate ignorance and variability, Reliab. Eng. Syst. Safe., 54, 133-144, 1996.

Freissinet, C., Vauclin, M., and Erlich, M.: Comparison of firstorder analysis and fuzzy set approach for the evaluation of imprecision in a pesticide groundwater pollution screening model, J. Contam. Hydrol., 37, 21-43, 1999.

Ghosh, S. and Mujumdar, P. P.: Climate change impact assessment: Uncertainty modeling with imprecise probability, J. Geophys. Res., 114, D18113, doi:10.1029/2008JD011648, 2009.

Hall, J. W.: Uncertainty-based sensitivity indices for imprecise probability distributions, Reliab. Eng. Syst. Safe., 91(10-11), 1443-1451, 2006.

Jacquin, A. P. and Shamseldin, A. Y.: Development of a possibilistic method for the evaluation of predictive uncertainty in rainfall-runoff modeling, Water Resour. Res., 43, W04425, doi:10.1029/2006WR005072, 2007.

Jacquin, A. P. and Shamseldin, A. Y.: Possibilistic uncertainty analysis of hydrological models, in: 8th International Conference on Hydroinformatics, Concepción, 12-16 January 2009, conf188a41, 2009.

Kamann, P. G.: Pronóstico estacional de deshielos en base a simulación, Memoria para Optar al Título de Ingeniero Civil, Universidad Técnica Federico Santa María, Chile, 1998.

Klir, G. J. and Folger, T. A.: Fuzzy sets, uncertainty and information, Prentice Hall, Singapore, 1992.

Klir, G. J. and Geer, J. F.: Information-preserving probabilitypossibility transformations: Recent developments, in: Fuzzy Logic, edited by: Lowen, R. and Roubens, M., Kluwer Academic Publishers, Dordrecht, 417-428, 1993.
Kuckzera, G.: On the validity of first order prediction limits for conceptual hydrologic models, J. Hydrol., 103, 229-247, 1988.

Lei, J. H. and Schilling, W.: Preliminary uncertainty analysis-a prerequisite for assessing the predictive uncertainty of hydrologic models, Water Sci. Technol., 33(2), 79-90, 1996.

Martin-Clouaire, R., Cazemier, D. R., and Lagacherie, P.: Representing and processing uncertain soil information for mapping soil hydrological properties, Comput. Electron. Agr., 29, 41-57, 2000.

Matott, L. S., Babendreier, J. E., and Purucker, S. T.: Evaluating uncertainty in integrated environmental models: A review of concepts and tools, Water Resour. Res., 45, W06421, doi:10.1029/2008WR007301, 2009.

Misirli, F., Gupta, H. V., Sorooshian, S., and Thiemann, M.: Bayesian recursive estimation of parameter and output uncertainty for watershed models, in: Calibration of Watershed Models, edited by: Duan, Q., Gupta, H., Sorooshian, S., Rousseau, A., and Turcotte, R. V., American Geophysical Union, Washington, 113-124, 2003.

Montanari, A.: Large sample behaviors of the generalized likelihood uncertainty estimation (GLUE) in assessing the uncertainty of rainfall-runoff simulations, Water Resour. Res., 41, W08406, doi:10.1029/2004WR003826, 2005.

Montanari, A., Shoemaker, C. A., and van de Giesen, N.: Introduction to special section on Uncertainty Assessment in Surface and Subsurface Hydrology: An overview of issues and challenges, Water Resour. Res., 45, W00B00, doi:10.1029/2009WR008471, 2009.

Mujumdar, P. P. and Ghosh, S.: Modeling GCM and scenario uncertainty using a possibilistic approach: Application to the Mahanadi River, India, Water Resour. Res., 44, W06407, doi:10.1029/2007WR006137, 2008.

Nash, J. E. and Sutcliffe, J. V.: River flow forecasting through conceptual models, Part I - A discussion of principles, J. Hydrol., 10, 282-290, 1970.

Nijssen, D., Schumann, A., Pahlow, M., and Klein, B.: Planning of technical flood retention measures in large river basins under consideration of imprecise probabilities of multivariate hydrological loads, Nat. Hazards Earth Syst. Sci., 9, 1349-1363, doi:10.5194/nhess-9-1349-2009, 2009.

Özelkan, E. C. and Duckstein, L.: Fuzzy conceptual rainfall-runoff models, J. Hydrol., 253, 41-68, 2001.

Pappenberger, F. and Beven, K.: Ignorance is bliss: Or seven reasons not to use uncertainty analysis, Water Resour. Res., 42, W05302, doi:10.1029/2005WR004820, 2006.

Renard, B., Kavetski, D., Kuczera, G., Thyer, M. A., and Franks, S. W.: Understanding predictive uncertainty in hydrologic modeling: The challenge of identifying input and structural errors, Water Resour. Res., 46, W05521, doi:10.1029/2009WR008328, 2010.

Rivera, A., Acuña, C., Casassa, G., and Bown, F.: Use of remotely sensed and field data to estimate the contribution of Chilean glaciers to eustatic sea-level rise, Ann. Glaciol. 34, 367-372, 2002.

Romanowicz, R., Beven, K., and Tawn, J. A.: Evaluation of predictive uncertainty in nonlinear hydrological models using a Bayesian approach, in: Statistics for the Environment 2: Water Related Issues, edited by: Barnett, V. and Feridun Turkman, K., John Wiley \& Sons, Chichester, 297-317, 1994. 
Rojas, R., Batelaan, O., Feyen, L., and Dassargues, A.: Assessment of conceptual model uncertainty for the regional aquifer Pampa del Tamarugal - North Chile, Hydrol. Earth Syst. Sci., 14, 171192, doi:10.5194/hess-14-171-2010, 2010.

Ross, T. J., Sellers, K. F., and Booker, J. M.: Considerations for using fuzzy set theory and probability theory, in: Fuzzy logic and probability applications: Building the gap, ASA-SIAM Series on Statistics and Applied Probability, edited by: Ross, T. J., Booker, J. M., and Parkinson, W. J., SIAM, Philadelphia, ASA, Alexandria, 87-104, 2002.

Shafer, G.: A Mathematical Theory of Evidence, Princeton University Press, Princeton, 1976.

Seibert, J.: Estimation of Parameter Uncertainty in the HBV Model, Nord. Hydrol., 28(4/5), 247-262, 1997.

Thiemann, M., Trosset, M., Gupta, H., and Sorooshian, S.: Bayesian recursive parameter estimation for hydrologic models, Water Resour. Res., 37(10), 2521-2535, 2001.

Thorsen, M., Refsgaard, J. C., Hansen, S., Pebesma, E., Jensen, J. B., and Kleeschulte, S.: Assessment of uncertainty in simulation of nitrate leaching to aquifers at catchment scale, J. Hydrol., 242, 210-227, 2001.

Valdivia, P.: Inventario de glaciares Andes de Chile central $\left(32^{\circ}-\right.$ $35^{\circ}$ Lat. S): Hoyas de los Ríos Aconcagua, Maipo, Cachapoal y Tinguiririca, in: Jornadas de Hidrología de Nieves y Hielos en América del Sur, PHI, UNESCO, Santiago, Chile, 3-8 December 1984, I6.1-I6.24, 1984.

Verhoest, N. E. C., De Baets, B., Mattia, F., Satalino, G., Lucau, C., and Defourny, P.: A possibilistic approach to soil moisture retrieval from ERS synthetic aperture radar backscattering under soil roughness uncertainty, Water Resour. Res, 43, W07435, doi:10.1029/2006WR005295, 2007.
Wagener, T., Wheater, H. S., and Gupta, H. V.: Identification and evaluation of watershed models, in: Calibration of Watershed Models, edited by: Duan, Q., Gupta, H. V., Sorooshian, S., Rousseau, A. N., and Turcotte, R., American Geophysical Union, Washington, 29-47, 2003.

Water Management Ltda.: Evaluación Recursos Hídricos Salar Punta Negra, Compañía Minera Escondida, Chile, 2001.

Wolkenhauer, O.: Possibility Theory with Applications to data analysis, John Wiley \& Sons, New York, 1998.

Yu, P.-S., Yang, T. C., and Chen, S. J.: Comparison of uncertainty analysis methods for a distributed rainfall-runoff model, J. Hydrol., 244, 43-59, 2001.

Zadeh, L. A.: Fuzzy Sets, Inform. Control, 8(3), 338-353, 1965.

Zadeh, L. A.: Fuzzy sets as a basis for a theory of possibility, Fuzzy Set. Syst., 1, 3-28, 1978.

Zadeh, L. A.: Possibility theory and soft data analysis, in: Mathematical Frontiers of the Social and Policy Sciences, edited by: Cob, B. L. and Thrall, R. M., Westview Press, Boulder, Colorado, 69-129, 1981.

Zadeh, L. A. and Desoer, C. A.: Linear System Theory: The State Space Approach, McGraw-Hill, New York, London, 1963.

Zehe, E. and Blöschl, G.: Predictability of hydrologic response at the plot and catchment scales: The role of initial conditions, Water Resour. Res., 40, W10202, doi:10.1029/2003WR002869, 2004.

Zhang, K., Li, H., and Achari, G.: Fuzzy-stochastic characterization of site uncertainty and variability in groundwater flow and contaminant transport through a heterogeneous aquifer, J. Contam. Hydrol., 106, 73-82, 2009.

Zimmermann, H.-J.: Fuzzy Set Theory and Its Applications, Fourth Edition, Kluwer Academic Publishers, Boston, 2001. 